\title{
Caracterização de Quitosanas Comerciais de Diferentes Origens
}

\author{
José E. dos Santos, João da P. Soares, Edward R. Dockal \\ Departamento de Química, UFSCar
}

\section{Sérgio P. Campana Filho, Éder T. G. Cavalheiro Instituto de Química de São Carlos, USP}

\begin{abstract}
Resumo: A quitosana é um polissacarídeo linear obtido da desacetilação da quitina. As propriedades físico-químicas da quitosana são função do grau médio de acetilacão $\overline{G A}$ e da massa molar média $(\bar{M})$. Várias aplicações da quitosana foram propostas na literatura, principalmente no tratamento de águas, produção de cosméticos, drogas e medicamentos, aditivos alimentícios, membranas semipermeáveis e no desenvolvimento de biomateriais. Neste trabalho, três diferentes tipos de quitosanas comerciais foram caracterizados por ressonância magnética nuclear de hidrogênio (RMN ${ }^{1} \mathrm{H}$ ), espectroscopia de absorção na região do infravermelho (IV), titulação condutimétrica e análise térmica (TG e DSC) com o objetivo de comparar as características de quitosanas provenientes de diferentes fornecedores. A massa molar média das amostras foi determinada a partir de medidas de viscosidade intrínseca. Os resultados obtidos por estas técnicas mostraram diferenças quanto ao grau médio de acetilação (ou desacetilação), em função da procedência do material. Os resultados obtidos da análise térmica permitiram acompanhar os processos de desidratação, decomposição e a temperatura de transição vítrea $\left(\mathrm{T}_{\mathrm{g}}\right)$.
\end{abstract}

Palavras-chave: Quitosana, grau médio de acetilação, termogravimetria, DSC.

\section{Characterization of commercial chitosan from different suppliers}

Abstract: Chitosan is a linear polysaccharide obtained from the deacetylation of chitin. The physico-chemical properties of chitosan dependent on the average degree of acetylation $(\overline{D A})$ and of the average molar mass $(\bar{M})$. Several applications of chitosan have been proposed in the literature, mainly in water treatment, cosmetic and drug manufacturing, food additives, semipermeable membranes and development of biomaterials. In this work, three commercial chitosan samples were characterized by proton nuclear magnetic resonance ( ${ }^{1} \mathrm{H}$ NMR), infrared spectroscopy (IR), conductimetric titration and thermal analysis (TG and DSC) in order to compare commercial chitosans from different supplies. The average viscosimetric molecular weight $\mathrm{M}_{\mathrm{v}}$ was estimated from the intrinsic viscosity. The results confirmed remarkable differences in relation to the mean degree of acetylation $(\overline{D A})$ (or desacetylation, $\overline{D D}$ ). The thermal analysis data permitted to follow the dehydration, decomposition, and glass transition temperature $(\mathrm{Tg})$.

Keywords: Chitosan, medium degree of acetylation, thermogravimetry, DSC.

\section{Introdução}

A quitosana é um biopolímero obtido da desacetilação da quitina, que é o maior constituinte de exoesqueletos de crustáceos e outros animais marinhos ${ }^{[1]}$. Atualmente a quitosana vem sendo utilizada no tratamento de águas, produção de cosméticos, drogas e medicamentos, aditivos alimentícios, membranas semipermeáveis e no desenvolvimento de biomateriais ${ }^{[2]}$. Na presença de soluções diluídas de ácidos, a quitosana comporta-se como um polieletrólito catiônico, constituído de um copolímero de 2-amino-2-deoxi-D-glicopiranose e 2-acetamido-2-deoxiD-glicopiranose de composição variável em função do grau médio de acetilação, $\overline{G A}$, que representa a fração de unidades 2-acetamido-2-glicopiranose e 2-amino-2-deoxi-D- glicopiranose e é um dos principais parâmetros para sua caracterização. A proporção relativa dessas unidades nas cadeias macromoleculares de quitosana tem efeito marcante na sua solubilidade. A representação de uma unidade de repetição da quitosana é dada na Figura 1.

Várias técnicas foram propostas para a determinação do $\overline{G A}$ de quitosana, baseadas em titulação condutimétrica ${ }^{[3]}$, espectroscopia na região do infravermelho (IV) ${ }^{[4]}$, espectroscopia de ressonância magnética de hidrogênio $\left(\operatorname{RMN~}^{1} \mathrm{H}\right)^{[5]}$, análise elementar ${ }^{[6]}$, cromatografia líquida de alta eficiência $(\mathrm{HPLC})^{[7]}$, termogravimetria (TG/DTG) ${ }^{[8]}$, entre outras.

A quitosana é insolúvel em água, mas dissolve-se em soluções aquosas de ácidos orgânicos, como acético, fórmico, cítrico, além de ácidos inorgânicos, como ácido clorídrico diluído resultando em soluções viscosas. 


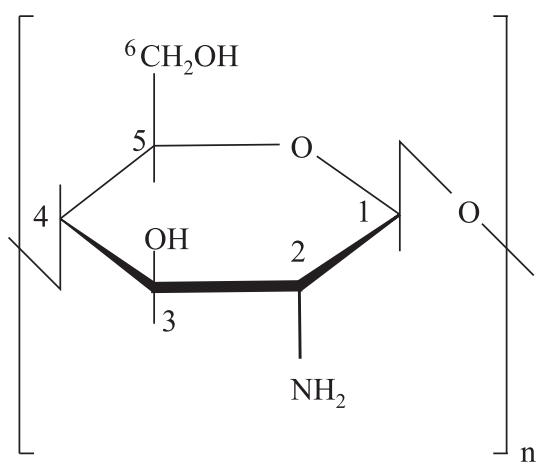

Figura 1. Fórmula estrutural do anel glicopiranosídico, a unidade de repetição monomérica da quitosana.

A solubilidade da quitosana está relacionada com a quantidade de grupos amino protonados $\left(-\mathrm{NH}_{3}{ }^{+}\right)$na cadeia polimérica. Quanto maior a quantidade destes grupos, maior a repulsão eletrostática entre as cadeias e também maior a solvatação em água. O grau de protonação pode ser determinado pela variação da concentração de quitosana. Para uma dada concentração de ácido, o grau de protonação depende do pK do ácido usado para solubilizar a quitosana ${ }^{[9]}$.

A quitosana é susceptível a mudanças estruturais, devido à grande quantidade de grupos reativos como as hidroxilas e principalmente os grupos amino, especialmente em reações de N-acetilação, N-alquilação, N-carboxilação, N-sulfonação e formação de bases de Schiff com aldeídos e cetonas ${ }^{[10]}$.

O objetivo deste trabalho é a caracterização de três amostras comerciais de diferentes marcas de quitosana e o estudo do comportamento térmico, usando termogravimetria (TG) e calorimetria exploratória diferencial (DSC). A caracterização é baseada no grau médio de acetilação $\overline{G A}$ e/ou grau médio de desacetilação $(\overline{G D})$, calculados por ressonância magnética nuclear de hidrogênio $\left(\mathrm{RMN}{ }^{1} \mathrm{H}\right)$ e titulação condutimétrica.

\section{Experimental}

\section{Reagentes}

Os reagentes e solventes utilizados neste trabalho foram de grau analítico (PA), e usados sem purificação prévia, exceto as amostras de quitosana comercial. Estas amostras foram obtidas das empresas Fluka ${ }^{\circledR}(\mathrm{QF})$, Aldrich ${ }^{\circledR}(\mathrm{QA})$ e Polymar/Br (QP). Todas elas, foram obtidas preliminarmente de exoesqueletos de carangueijo. Segundo especificação dos fabricantes, as amostras QF e QP apresentam massa molar média e nenhuma especificação quanto ao $\overline{G D}$, enquanto a amostra QA apresenta massa molar baixa e $\overline{G D}$ de $84,7 \%$.

\section{Purificação das amostras de quitosana}

As amostras de quitosana foram purificadas de acordo com o procedimento descrito por Signini e Campana-Filho ${ }^{[11]}$. Amostra de quitosana foi dissolvida em ácido acético $0,5 \mathrm{~mol} \mathrm{~L}^{-1}$ sob agitação constante por aproximadamente 18 horas. A solução resultante foi filtrada sob pressão positiva através de membranas de porosidades 5 e $0,8 \mu \mathrm{m}$. À solução filtrada adicionou-se aos poucos hidróxido de amônio concentrado até a precipitação. O precipitado foi lavado com água várias vezes até $\mathrm{pH}$ neutro, em seguida lavado com metanol seco a temperatura ambiente e depois em estufa a vácuo, a $60{ }^{\circ} \mathrm{C}$ por 24 horas.

\section{Caracterização das Amostras}

\section{Espectroscopia na região do Infravermelho (IV)}

Aproximadamente $1,5 \mathrm{mg}$ das amostras de quitosana foram secas em estufa a vácuo por 15 horas a $60^{\circ} \mathrm{C}$. Após este período, $100 \mathrm{mg}$ de $\mathrm{KBr}$ foram adicionados e a mistura homogeneizada em almofariz de ágata. As pastilhas foram preparadas e deixadas em estufa a vácuo a $110^{\circ} \mathrm{C}$ por 20 horas. Os espectros de IV foram registrados em um espectrofotômetro Bomem-Michelson FT-IR, MB 102.

\section{Ressonância Magnética Nuclear de Hidrogênio (RMN $\left.{ }^{1} H\right)$}

Os espectros de $\mathrm{RMN}{ }^{1} \mathrm{H}$ foram obtidos em um espectrômetro BRUKER DRX400. Todos os espectros foram obtidos a partir de um procedimento descrito por Signini e Campana-Filho ${ }^{[12]}$. Aproximadamente $10 \mathrm{mg}$ de amostra de quitosana foi solubilizada em $1 \mathrm{~mL}$ de solução de $\mathrm{HCl} / \mathrm{D}_{2} \mathrm{O}$ $1 \%(\mathrm{v} / \mathrm{v})$, durante 24 horas formando uma solução viscosa. Uma alíquota dessa solução foi colocada em tubos de $5 \mathrm{~mm}$ de diâmetro para a análise a $70^{\circ} \mathrm{C}$. Como referência externa foi usado ácido 3-(trimetilsilil)-1-propano sulfonico-d4 (TSPA da Aldrich).

\section{Titulação condutimétrica}

O procedimento foi adaptado do trabalho de Raymond et al. ${ }^{[3]}$. Neste caso, $200 \mathrm{mg}$ das amostras de quitosana foram agitadas em $40 \mathrm{~mL}$ de solução de ácido clorídrico $0,05 \mathrm{~mol} \mathrm{~L}^{-1}$ por 18 horas. As amostras foram tituladas com solução por $\mathrm{NaOH} 0,17 \mathrm{~mol} \mathrm{~L}^{-1}$ à temperatura de $25,0^{\circ} \pm 0,1^{\circ} \mathrm{C}$. As variações de condutância durante a titulação foram medidas por um condutivímetro Micronal B330, equipado com célula condutimétrica Digimed DMC-010.

\section{Viscosidade}

Para determinações de viscosidade intrínseca, [ๆ], foi adotado o procedimento descrito por Signini e Campana Filho ${ }^{[12]}$. Cerca de $50 \mathrm{mg}$ de amostra de quitosana purificada foi dissolvida em $25 \mathrm{~mL}$ de uma solução tampão (ácido acético / acetato de sódio, $\mathrm{pH} \sim 4,5)$ sob agitação constante por 15 horas. A solução foi então aquecida em banho-maria a $80^{\circ} \mathrm{C}$ durante dois minutos e após atingir a temperatura ambiente, acrescentou-se $25 \mathrm{~mL}$ de solução tampão e aqueceu-se novamente a $80{ }^{\circ} \mathrm{C}$ por dois minutos. Após atingir a temperatura ambiente, a solução foi filtrada em membrana de porosidade 0,45 $\mu \mathrm{m}$. Alíquota de $15 \mathrm{~mL}$ foi colocada em capilar de vidro $(\phi=0,45 \mathrm{~mm})$ termostatizado em $25^{\circ} \pm 0,1^{\circ} \mathrm{C}$ para diluição seriada. Os tempos de escoamento foram determinados em viscosímetro AVS-350 acoplado ao sistema diluidor automático AVS 20, ambos da Schott-Geräte. A solução, com viscosidade relativa menor que 1,8 , foi seqüencialmente diluída 
no próprio capilar através da adição do tampão ácido acético/ acetato $(\mathrm{pH}=4,5)$, até atingir uma viscosidade relativa próxima de 1,2 . Os valores de tempos de escoamento de [ $\eta]$ correspondem à média de quatro determinações independentes e que não apresentaram variação maior que $0,09 \%$. Todos os tempos de escoamento foram automaticamente corrigidos para efeitos cinéticos pelo acionamento de dispositivo específico no sistema de gerenciador das medidas.

\section{Análise Térmica}

As curvas TG foram obtidas em um módulo termogravimétrico TGA 2050 e as DSC em um módulo calorimétrico DSC 2100, acoplados a um analisador térmico TA 2000, todos da TA Instruments.

As medidas termogravimétricas foram efetuadas usando suporte de amostra de platina, massas de amostra em torno de $7 \mathrm{mg}$, razão de aquecimento de 5, 10, 20 e $30^{\circ} \mathrm{C} \mathrm{min}^{-1} \mathrm{em}$ atmosfera dinâmica de ar e $\mathrm{N}_{2}$, ambas com uma vazão de 90 $\mathrm{mL} \mathrm{min}^{-1}$.

As curvas DSC foram obtidas em suporte de amostra de alumínio com tampa furada no centro e massas de amostra em torno de $4 \mathrm{mg}$. As amostras foram aquecidas a uma razão de aquecimento de $10{ }^{\circ} \mathrm{C} \mathrm{min}^{-1} \mathrm{em}$ atmosfera dinâmica de ar e $\mathrm{N}_{2}$, ambas com uma vazão de $90 \mathrm{~mL}$ min $^{-1}$.

\section{Resultados e Discussão}

\section{Espectroscopia na região do infravermelho}

O espectro de absorção no infravermelho (IV) da amostra QF é apresentado na Figura 2, como exemplo representativo dos demais, já que todos foram semelhantes.

As principais bandas observadas nos espectros IV das amostras de quitosana foram: banda de estiramento axial de $\mathrm{OH}$ entre $3440 \mathrm{a} 3480 \mathrm{~cm}^{-1}$, a qual aparece sobreposta à banda de estiramento $\mathrm{N}-\mathrm{H}$; deformação axial de $\mathrm{C}=\mathrm{O}$ de amida I (entre 1661 a $1671 \mathrm{~cm}^{-1}$ ); deformação angular de N-H (entre 1583 a $1594 \mathrm{~cm}^{-1}$ ); deformação angular simétrica de $\mathrm{CH}_{3}$ (entre 1380 a $1383 \mathrm{~cm}^{-1}$ ); deformação axial de -CN de amida (por volta de $1425 \mathrm{~cm}^{-1}$ ) e deformação axial de -CN de grupos amino (entre 1308 a $1380 \mathrm{~cm}^{-1}$ ), além de bandas de estruturas polissacarídicas na região de $890-1156 \mathrm{~cm}^{-1}$. Todas as bandas características são muito semelhantes aquelas descritas por Brugnerotto et $a l^{[4]}$ e estão presentes em todas as amostras

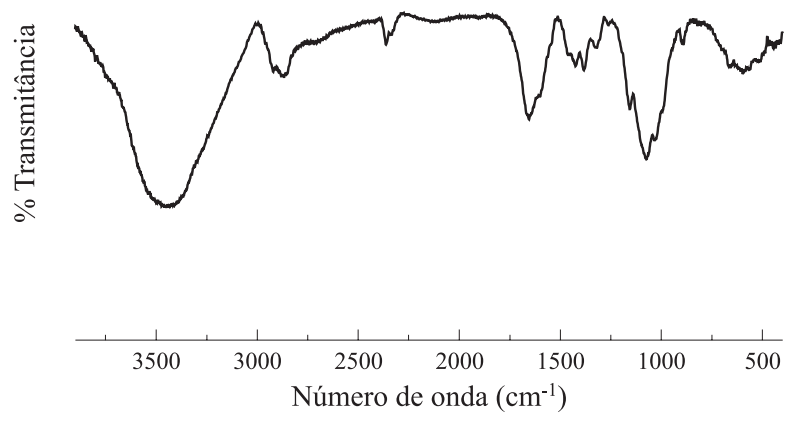

Figura 2. Espectro de absorção na região do infravermelho(IV) da amostra QF. comerciais investigadas, mostrando que todas apresentam basicamente os mesmos grupos funcionais.

\section{Grau médio de acetilação ou desacetilação}

O grau médio de desacetilação $(\overline{G D})$ é definido como o número de grupos amino em relação aos grupos amida da cadeia polimérica, podendo ser determinado por meio de várias técnicas ${ }^{[3-8]}$. No presente caso, descreveu-se os resultados obtidos por condutimetria e RMN ${ }^{1} \mathrm{H}$, que fornece $\mathrm{o}$ grau médio de acetilação.

As curvas condutimétricas das amostras de quitosana usadas neste trabalho com solução de $\mathrm{NaOH}$ e excesso de ácido clorídrico são mostradas na Figura 3. O primeiro ramo linear representa a neutralização do ácido presente, o segundo corresponde à neutralização de prótons dos grupos amino da quitosana e o terceiro conjunto de pontos refere-se ao excesso de base, após o ponto de equivalência. Estas três retas originam por extrapolação dois pontos de inflexão, que correspondem ao volume de base necessário para neutralizar os grupos amino protonados. O número de equivalentes de grupos ácidos foi calculado usando a Equação 1.

$$
\% \overline{G D}=\frac{16,1 \cdot[\text { base }] .\left(V_{2}-V_{1}\right)}{m}
$$

$$
\% \overline{G A}=100-\% \overline{G D}
$$

Nestas equações, $\overline{G D}$ é o grau médio de desacetilação, $\overline{G A}$ é o grau médio de acetilação, $\mathrm{V}_{1}$ é o volume de base usado para a neutralização de $\mathrm{HCl}$ em excesso $(\mathrm{mL}) ; V_{2}-V_{I}$ é o volume de base usado para a neutralização dos grupos

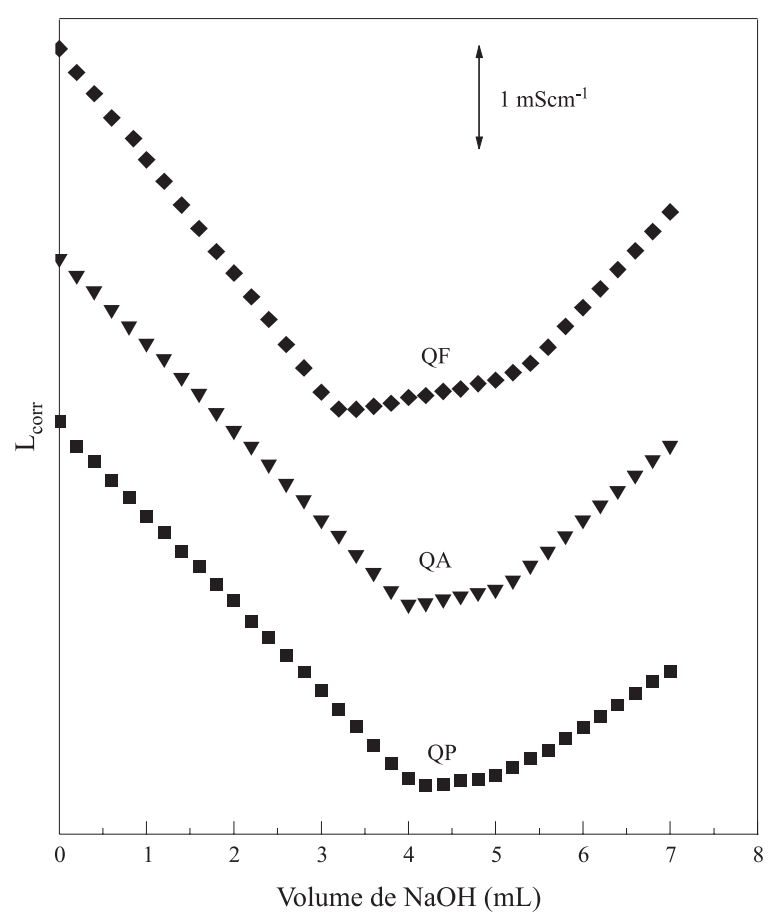

Figura 3. Curva de titulação condutimétrica das amostras de quitosana (QP, QA e QF). 
Tabela 1. Valores de $\overline{G D}$ determinados por titulação condutimétrica e por RMN ${ }^{1} \mathrm{H}$ em comparação aos valores rotulados.

\begin{tabular}{|c|c|c|c|c|}
\hline \multirow{2}{*}{ Amostra } & \multicolumn{3}{|c|}{$\% \overline{G D}$} & \multirow{2}{*}{$|\mathbf{E R}|^{\mathrm{a}} / \%$} \\
\hline & $\begin{array}{c}\% \overline{G D} \\
\text { rotulado }\end{array}$ & $\mathbf{R M N}^{1} \mathrm{H}$ & Condutimetria & \\
\hline QF & $\begin{array}{c}\text { não } \\
\text { especificado }\end{array}$ & 77,0 & 74,8 & 2,8 \\
\hline QA & 84,7 & 90,2 & 89,9 & 0,4 \\
\hline $\mathrm{QP}$ & $\begin{array}{c}\text { não } \\
\text { especificado }\end{array}$ & 90,0 & 89,6 & 0,4 \\
\hline
\end{tabular}

a(Ressonância - Condutimetria / Ressonância) x 100

ácidos de quitosana $(\mathrm{mL})$; [base] é a concentração da base usada e $m$ é a massa da amostra de quitosana.

$\mathrm{Na}$ Tabela 1, estão apresentados os valores de grau médio de desacetilação $\overline{G D}$ das amostras QF, QA e QP, determinados por titulação condutimétrica usando a Equação 1, e o grau médio de acetilação $\overline{G A}$ por $\mathrm{RMN}{ }^{1} \mathrm{H}$ calculado com auxílio da Equação 3.

Como foi feito para o infravermelho, apresenta-se na Figura 4 o espectro de $\mathrm{RMN}{ }^{1} \mathrm{H}$ da amostra $\mathrm{QA}$, como exemplo representativo, dado a sua semelhança com os demais. A espectroscopia de $\mathrm{RMN}{ }^{1} \mathrm{H}$ é quantitativa nas análises de amostras de quitosana em relação aos valores de $\overline{G A}$. Como a dissolução da quitosana em meio ácido resulta em uma solução viscosa, faz-se necessário que a medida seja realizada a $70{ }^{\circ} \mathrm{C}$. No entanto, é necessário que a varredura do espectro e a aquisição de dados sejam realizadas rapidamente ao se efetuar a análise nesta temperatura, de modo a minimizar problemas causados pela hidrólise ácida ${ }^{[13]}$. A determinação de grau médio de acetilação $\overline{G A}$ por $\mathrm{RMN}{ }^{1} \mathrm{H}$ das amostras investigadas foi feita utilizando a área do pico na região de 2 ppm atribuído aos núcleos de hidrogênio da metila do grupo acetamido $\left(A_{C H 3}\right)$ e a área do pico em 3,2 ppm atribuído ao núcleo de hidrogênio na posição 2 do anel de glicosamino $\left(A_{H 2}\right)$, segundo procedimento descrito por Signini e Campana-Filho ${ }^{[12]}$.

$$
\% \overline{G A}=\left(\frac{A_{C H 3}}{3 A_{H 2}}\right) x 100
$$

A escolha da relação de áreas representada pela Equação 3, se deve ao fato de que as áreas relativas a núcleos dos grupos metila presentes no grupo acetamido e ao núcleo na posição

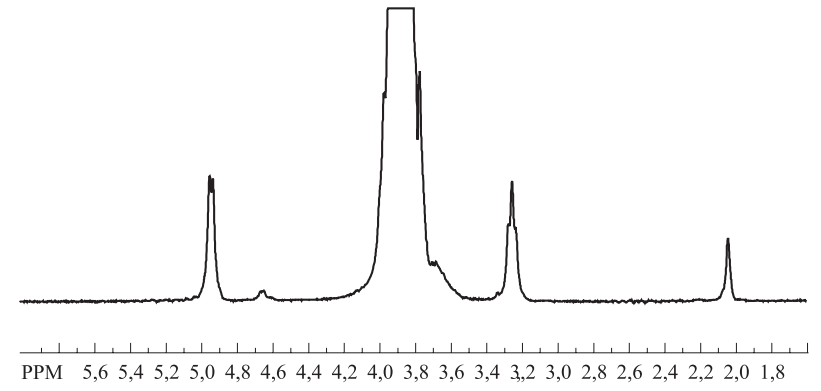

Figura 4. Espectro de RMN ${ }^{1} \mathrm{H}$ da amostra $\mathrm{QA}$ a $70^{\circ} \mathrm{C}$.
Tabela 2. Análise elementar de amostras de quitosana comerciais

\begin{tabular}{cccc}
\hline Amostra & \% $\mathbf{C}$ & $\mathbf{\% H}$ & $\boldsymbol{\%} \mathbf{N}$ \\
\hline QA & 43,5 & 6,5 & 7,9 \\
QF & 44,5 & 6,7 & 8,5 \\
QP & 41,2 & 6,0 & 7,0 \\
\hline
\end{tabular}

2 do anel de glicosamino, estão relativamente livres das influências do pico de $\operatorname{HOD}(\delta=3,8 \mathrm{ppm})$.

A determinação do grau médio de desacetilação $\overline{G D}$ por titulação condutimétrica é um método simples, de baixo custo e não destrutivo, que apresentou concordância com os valores de $\overline{G D}$ obtidos por RMN ${ }^{1} \mathrm{H}$. Os fabricantes não fornecem informações a respeito dos valores de $\overline{G D}$, logo, é importante comparar métodos que permitam determinar este parâmetro para prever propriedades físico-químicas da quitosana, oferecendo opções quanto a custo e grau de dificuldade experimental de diferentes técnicas.

\section{Análise elementar}

Os dados de análise elementar apresentados na Tabela 2 são próximos da fórmula $\mathrm{C}_{6} \mathrm{H}_{11} \mathrm{O}_{4} \mathrm{~N}$ para a unidade de repetição, quando se desconta o teor de água presente na amostra. O teor de água pode ser obtido por termogravimetria, como descrito abaixo.

A hidratação se deve ao fato de que a quitosana tem forte afinidade pela água e, no estado sólido, as macromoléculas podem apresentar estruturas distorcidas, que são facilmente hidratadas ${ }^{[14]}$. Em adição, deve-se considerar que a análise elementar é um método conveniente para análise de quitosana somente na ausência de proteína residual ${ }^{[4]}$.

\section{Viscosidade}

Na Tabela 3 são apresentados os valores de grau médio de acetilação, $\overline{G A}$, de viscosidade intrínseca, $([\eta])$, e os valores encontrados da massa molar média viscosimétrica $\left(M_{V}\right)$ para as amostras de quitosana (QA e QF). Não foi possível realizar os estudos viscosimétricos com a amostra $Q P$, em virtude da baixa solubilidade da mesma.

A viscosimetria, embora seja um método não absoluto, é um dos processos mais utilizados para a determinação da massa molar de polímeros. As medidas são feitas com base no tempo de escoamento do solvente e das soluções diluídas do polímero, utilizando-se um viscosímetro. A viscosidade de uma

Tabela 3. Valores de viscosidade intrínseca ([ๆ]) e massas molares médias viscosimétricas $\left(M_{v}\right)$ de amostras de quitosanas comerciais.

\begin{tabular}{cccc}
\hline Amostra & $\boldsymbol{\%} \overline{\boldsymbol{G A}}$ & {$[\eta]\left(\mathbf{m L ~ \mathbf { g } ^ { - 1 } )}\right.$} & $\mathbf{M}_{\mathbf{v}} \times \mathbf{x 1 0}^{\mathbf{4}}\left(\mathbf{g ~ m o l}^{-\mathbf{1}}\right)$ \\
\hline QA & 9,8 & 360 & $6,9^{(\mathrm{a})}$ \\
QF & 23,0 & 942 & $25,2^{(\mathrm{b})}$ \\
\hline
\end{tabular}

(a) valor calculado empregando $\mathrm{K}=0,076$ e $\alpha=0,76^{[16]}$; (b) valor calculado empregando $\mathrm{K}=0,074$ e $\alpha=0,76^{[16]} \mathrm{em}$ solvente $\mathrm{NaAc} 0,2 \mathrm{molL}^{-1} / \mathrm{HAc}$ $0,3 \mathrm{molL}^{-1}$. 


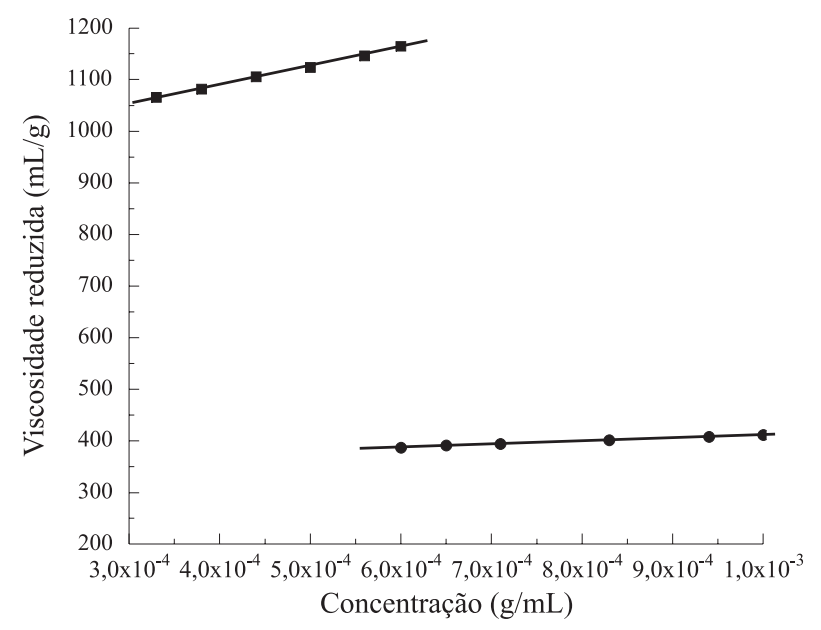

Figura 5. Curvas de viscosidade reduzida versus concentração das amostras de quitosana comercial ( $\square$ QA e $\bullet$ QF) purificada em tampão NaAc/ $\mathrm{HAc} \mathrm{pH}=4,5$.

solução de polieletrólito pode ser descrita como função de sua viscosidade intrínseca e de sua concentração se não ocorrerem interações entre as macromoléculas (sistema diluído). Neste caso, a equação de Huggins ${ }^{[15]}$ pode ser usada:

$$
\frac{\eta_{s p}}{C}=[\eta]+\mathrm{K} \quad[\eta]^{2} \mathrm{C}
$$

em que: $\eta_{s p}$ é a viscosidade específica; $\eta_{\mathrm{sp}} / C$ é a viscosidade reduzida $\left(\mathrm{mLg}^{-1}\right) ;[\eta]$ é a viscosidade intrínseca $\left(\mathrm{mLg}^{-1}\right) ; K_{H}$

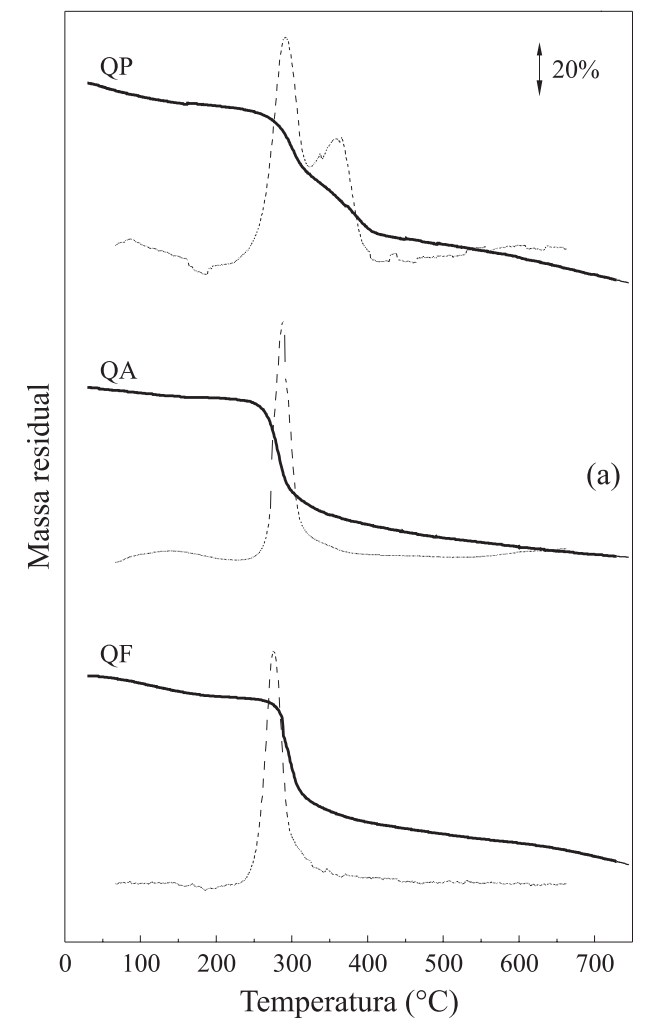

é a constante de Huggins e $C$ é a concentração da solução $\left(\mathrm{gmL}^{-1}\right)$

A Figura 5 mostra as curvas de viscosidade reduzida $\left(\eta_{\text {red }}\right)$ versus concentração da solução de quitosana das amostras QA e QF. A viscosidade intrínseca, [ $\eta$ ], foi determinada pela extrapolação dos dados de viscosidade à diluição infinita, de acordo com a equação 4. As retas obtidas apresentam excelentes coeficientes de correlação entre os pontos experimentais $(r>0,9)$ e os valores de constantes de Huggins, determinados no tampão ácido acético/acetato, são relativamente pequenos (Tabela 3), indicando que soluções de boa qualidade foram obtidas pela dissolução das amostras purificadas de quitosana. A viscosidade assim determinada satisfaz a relação de Mark-Houwink (Equação 5)

$$
[\eta]=\mathrm{K} \cdot \mathrm{M}_{\mathrm{V}}{ }^{\alpha}
$$

em que, $[\eta]$ é a viscosidade intrínseca da solução, $K$ é uma constante característica do polímero e depende da temperatura e do solvente, $\alpha$ é a constante característica da geometria da molécula do polímero e $\mathrm{M}_{\mathrm{v}}$ é a massa molecular viscosimétrica.

O valor de viscosidade intrínseca determinado a partir da Eq.5 proposta por Rinaudo et al ${ }^{[16]}$ permite determinar a massa viscosimétrica, $\mathrm{M}_{\mathrm{v}}$ das amostras QA e QF (Tabela 3).

\section{Termogravimetria (TG)}

Inicialmente, foi feita uma otimização da razão de aquecimento a ser usada nos estudos de decomposição térmica,

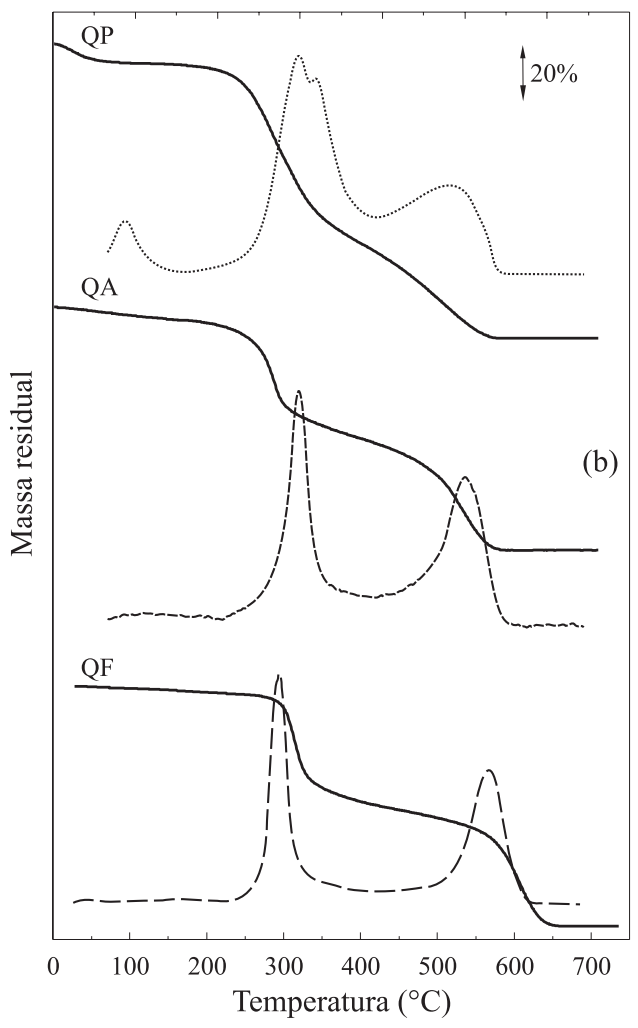

Figura 6. Curvas TG para as amostras de quitosanas comerciais sob atmosfera dinâmica (vazão de gás $90 \mathrm{~mL} \mathrm{~min}^{-1}$ ) de $\mathrm{N}_{2}$ (a) e ar (b). Massa de amostra aproximadamente $7 \mathrm{mg}$; razão de aquecimento $10^{\circ} \mathrm{C} \mathrm{min}^{-1}$; suporte de amostra de platina. 
usando a amostra QF, como modelo. A amostra foi submetida às razões de aquecimento de $5,10,20 \mathrm{e} 30^{\circ} \mathrm{C} \mathrm{min}^{-1}$. Tal avaliação foi necessária, pois a razão de aquecimento apresenta influência significativa no aspecto qualitativo da curva $\mathrm{TG}^{[17]}$, pois razões de aquecimento maiores levam uma menor definição e poderiam induzir a uma sobreposição de eventos térmicos sucessivos. Já razões menores tornam o experimento demorado.

Apesar da semelhança nos perfis nas curvas TG, concluiuse que a razão de $10{ }^{\circ} \mathrm{C} \mathrm{min}{ }^{-1}$ seria mais adequada para estudos envolvendo as quitosanas, devido ao perfil mais bem definido das curvas em relação aos $20^{\circ} \mathrm{C} \min ^{-1} \mathrm{e}$ ao menor tempo de análise em relação aos $5^{\circ} \mathrm{C} \min ^{-1}$.

Também foi desenvolvido um estudo da influência do gás de purga na decomposição térmica, para todas as amostras de quitosanas comerciais, usando atmosferas dinâmicas de ar e nitrogênio. Os resultados são apresentados na Figura $6 \mathrm{a}$ e $6 \mathrm{~b}$.

Tais experimentos revelaram que sob atmosfera dinâmica $\mathrm{N}_{2}$ (Fig. 6a), as amostras de QF, QA e QP apresentaram um

Tabela 4. Resultados de TG e DSC para a decomposição térmica das amostras de quitosana comercial em atmosfera de ar e nitrogênio

\begin{tabular}{|c|c|c|c|}
\hline \multirow[b]{2}{*}{ Evento Térmico } & \multicolumn{2}{|c|}{ TG } & \multirow[b]{2}{*}{ Picos DSC $/{ }^{\circ} \mathrm{C}$} \\
\hline & $\begin{array}{c}\text { Intervalo de } \\
\text { Temperatura/ } /{ }^{\circ} \mathrm{C}\end{array}$ & $\begin{array}{c}\text { Perda de } \\
\text { Massa }^{\mathrm{a}} \text { ou } \\
\text { Resíduo }^{\mathrm{b}} / \%\end{array}$ & \\
\hline \multicolumn{4}{|l|}{ Atmosfera de $N^{2}$} \\
\hline $\mathrm{QF} . \mathrm{nH}_{2} \mathrm{O} \rightarrow \mathrm{QF}+\mathrm{nH}_{2} \mathrm{O}$ & $25-228^{a}$ & 8,1 & 75,8 (endo) \\
\hline $\mathrm{QF} \rightarrow \mathrm{RC}^{\mathrm{c}}$ & $228-862^{b}$ & $18,78 \mathrm{~b}$ & 310,6 (exo) \\
\hline QA.nH ${ }_{2} \mathrm{O} \rightarrow \mathrm{QA}+\mathrm{nH}_{2} \mathrm{O}$ & $21-178,9^{\mathrm{a}}$ & 5,1 & 100,8 (endo) \\
\hline $\mathrm{QA} \rightarrow \mathrm{RC}^{\mathrm{c}}$ & $178,9-862^{b}$ & $18,48 \mathrm{~b}$ & 310,6 (exo) \\
\hline $\mathrm{QP} . \mathrm{nH}_{2} \mathrm{O} \rightarrow \mathrm{QP}+\mathrm{nH}_{2} \mathrm{O}$ & $29-175^{a}$ & 7,8 & 83,5 (endo) \\
\hline $\mathrm{QP} \rightarrow \mathrm{RC}^{\mathrm{c}}$ & $175-862 b$ & $17,73 b$ & 310 (exo) \\
\hline \multicolumn{4}{|l|}{ Atmosfera de ar } \\
\hline $\mathrm{QF} . \mathrm{nH}_{2} \mathrm{O} \rightarrow \mathrm{QF}+\mathrm{nH}_{2} \mathrm{O}$ & $30-214$ & $5,0 \mathrm{a}$ & 71,8 (endo) \\
\hline $\mathrm{QF} \rightarrow \mathrm{RC}^{\mathrm{c}}$ & $214-385,6$ & $46,0 \mathrm{a}$ & 309 (exo) \\
\hline Queima do RC & $385,6-700$ & $0,0 \mathrm{~b}$ & \\
\hline QA.nH ${ }_{2} \mathrm{O} \rightarrow \mathrm{QA}+\mathrm{nH}_{2} \mathrm{O}$ & $21-175,0$ & $5,0 \mathrm{a}$ & 71,3 (endo) \\
\hline $\mathrm{QA} \rightarrow \mathrm{RC}^{\mathrm{c}}$ & $175,0-372$ & $46,0 \mathrm{a}$ & 308,7 (exo) \\
\hline Queima do RC & $372-700$ & $0,0 \mathrm{~b}$ & \\
\hline $\mathrm{QP} . \mathrm{nH}_{2} \mathrm{O} \rightarrow \mathrm{QP}+\mathrm{nH}_{2} \mathrm{O}$ & $25,1-165,3$ & $7,7 \mathrm{a}$ & 73,4 (endo) \\
\hline $\mathrm{QP} \rightarrow \mathrm{RC}^{\mathrm{c}}$ & $165,3-388,6$ & $57,4 \mathrm{a}$ & 307,8 (exo) \\
\hline Queima do RC & $388,6-700$ & $0,1 \mathrm{~b}$ & \\
\hline
\end{tabular}

aperda de massa; ${ }^{b}$ relativo ao resíduo; 'resíduo carbonizado; exo $=$ exotérmico; endo $=$ endotérmico processo de desidratação, seguido da decomposição do biopolímero, com geração de material carbonizado. A decomposição deste material não se completa mesmo acima de $700{ }^{\circ} \mathrm{C}$. Sob ar, além dos eventos acima, observa-se a queima do material carbonizado formado na segunda etapa, sem resíduos no suporte de amostra já em torno de $600{ }^{\circ} \mathrm{C}$ (Fig. 6b).

As perdas de massa, percentagem de resíduos e intervalo de temperatura, observados em cada etapa das curvas de TG das amostras de quitosanas comerciais, são resumidos na Tabela 4.

Observa-se também que sob as mesmas condições experimentais, a amostra QF, com massa molar média, é a que apresenta maior estabilidade térmica após desidratação. Já as amostras QP e QA apresentaram estabilidade térmica semelhantes, apesar das diferenças de massa molar das cadeias poliméricas especificada pelos fabricantes. Assim seria possível considerar uma relação inversamente proporcional entre estabilidade térmica e grau de desacetilação, pois a amostra QF apresenta $\overline{G D}$ da ordem de $75 \%$, enquanto as amostras QA e QP apresentam um $\overline{G D}$ da ordem de $90 \%$.

\section{Calorimetria Exploratória Diferencial (DSC)}

A Figura 7a, mostra as curvas DSC de amostras de quitosanas comerciais sob atmosfera de $\mathrm{N}_{2}$ na faixa de temperatura entre 20 e $500{ }^{\circ} \mathrm{C}$. Observaram-se dois picos que são descritos na Tabela 4. O primeiro, endotérmico, correspondente ao processo de desidratação, cuja área depende do histórico de secagem da amostra e o segundo, exotérmico, corresponde ao processo de decomposição, que continua além do limite de temperatura do experimento. Os dois processos estão coerentes com os eventos observados nas curvas TG e concordam com o que foi observado por Sreenivasan ${ }^{[18]}$ que descreveu dois eventos térmicos para amostras de quitosana, sob $\mathrm{N}_{2}$ em curvas DSC.

A Figura 7b mostra curvas DSC de quitosanas comercias (QF, QA e QP) sob atmosfera dinâmica de ar na faixa de temperatura entre 24 e $500{ }^{\circ} \mathrm{C}$. Observaram-se três eventos térmicos, sendo o primeiro endotérmico correspondente ao processo de desidratação. O segundo pico, exotérmico, corresponde ao processo de decomposição. O terceiro evento corresponde ao início da queima do material carbonizado. Os três processos estão coerentes com os eventos observados no TG e são representados na Tabela 4.

Observa-se que o segundo processo de decomposição da amostra QP apresenta-se dividido em dois e com perfil térmico diferente das demais, em atmosfera de ar. Isso pode evidenciar a presença de materiais, tais como proteínas residuais, que não podem ser eliminados pelo processo de purificação utilizado.

A Figura 8 mostra curvas DSC de amostras de quitosana em temperatura sub-ambiente na faixa de -50 e $150^{\circ} \mathrm{C}$. A presença de temperatura de transição vítrea $\left(\mathrm{T}_{\mathrm{g}}\right)$ pode ser observada em aproximadamente $30^{\circ} \mathrm{C}$ para as amostras QA e QF e em torno de $10^{\circ} \mathrm{C}$ para a amostra QP, ainda que com pouca definição. Segundo Hatakeyama e Quinn ${ }^{[19]}$ 

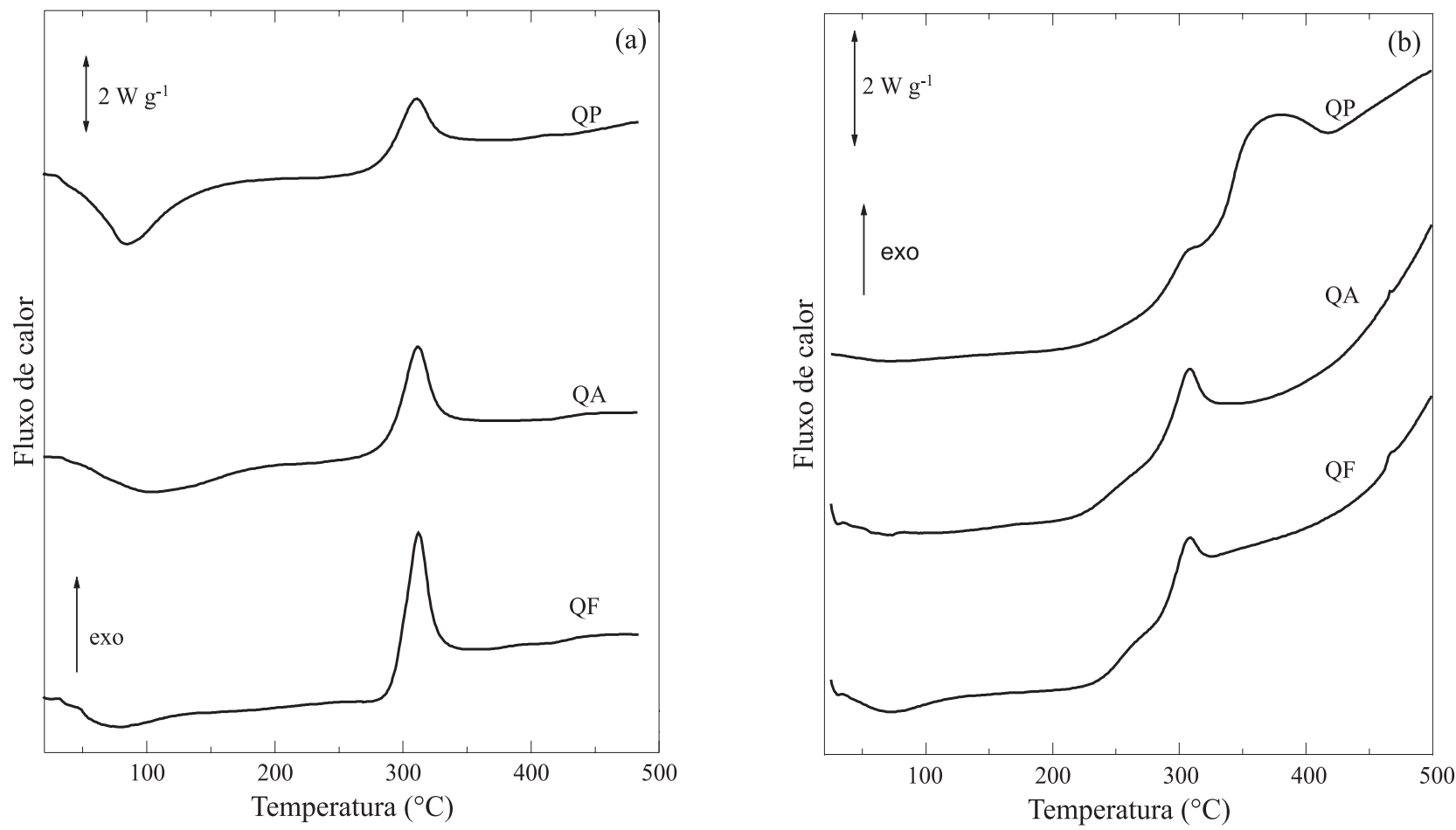

Figura 7. Curvas DSC para as amostras de quitosanas comerciais sob atmosfera dinâmica (vazão de gás $90 \mathrm{~mL} \mathrm{~min}^{-1}$ ) de $\mathrm{N}_{2}$ (a) e ar (b). Massa de amostra de aproximadamente $4 \mathrm{mg}$; razão de aquecimento $10{ }^{\circ} \mathrm{C} \mathrm{min}^{-1}$; suporte de amostra de alumínio com tampa furada no centro.

este fenômeno se torna difícil de visualizar por DSC, uma vez que o evento depende do teor de água presente na amostra.

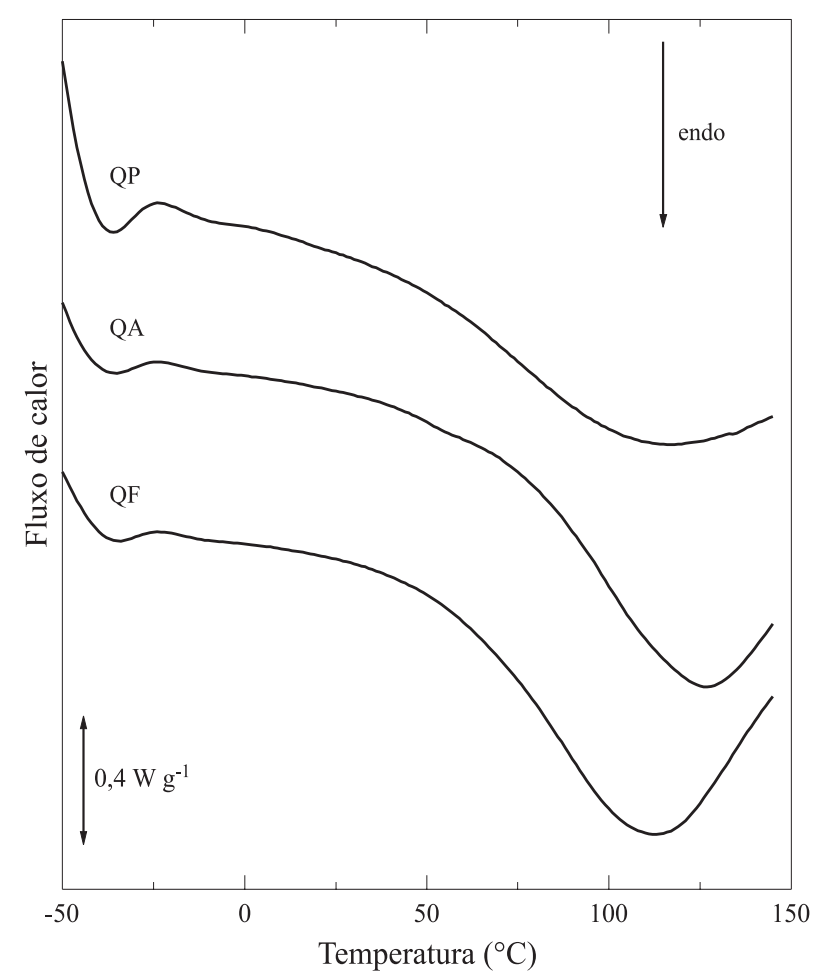

Figura 8. Curvas DSC iniciadas em temperatura sub-ambiente para as amostras de quitosanas comerciais sob atmosfera dinâmica de nitrogênio, massa de $11 \mathrm{mg}$ e razão de aquecimento $20^{\circ} \mathrm{C} \mathrm{min}^{-1}$, suporte de amostra de alumínio com tampa furada no centro.

\section{Conclusões}

A titulação condutimétrica e a ressonância magnética nuclear $\left(\mathrm{RMN}{ }^{1} \mathrm{H}\right)$ constituem métodos eficientes e reprodutíveis para determinar o grau médio de desacetilação ( ), embora a titulação condutimétrica tenha ligeira vantagem, por ser uma técnica não destrutiva e de baixo custo. Porém, a análise por $\mathrm{RMN}{ }^{1} \mathrm{H}$ requer preparação menos exigente amostra, devido à especificidade do pico do grupo acetamida.

Observou-se também que o $\overline{G D}$ varia dependendo do fabricante e não concordam exatamente com o valor rotulado, quando este é fornecido.

A análise elementar, apesar de ser um método preciso, deve ser usado com muita cautela em virtude dos diferentes teores de hidratação, que variam de acordo com as condições de armazenamento e tratamento prévio da amostra.

As técnicas termoanalíticas fornecem informações sobre o mecanismo de decomposição e estabilidade térmica, sendo a DSC mais apropriada para investigação da transição vítrea. Entretanto ela é fortemente dependente do teor de $\mathrm{H}_{2} \mathrm{O}$ enquanto a TG fornece informações a temperaturas mais elevadas, tendo sido observadas diferenças de estabilidade térmica nas amostras comerciais com diferentes massas moleculares e $\overline{G D}$.

As técnicas utilizadas mostraram diferenças em relação às características das quitosanas comerciais avaliadas. Entretanto, é preciso determinar o $\overline{G D}$ para caracterizar o material, não bastando confiar na especificação rotulada.

\section{Agradecimentos}

Os autores agradecem ao CNPq e a CAPES pelo apoio financeiro outorgado durante a realização deste trabalho. 


\section{Referências Bibliográficas}

1. Matteus, F.A.G(ed.). -“Application of Chitin and Chitosan”, Technomic Publishing AG, Switzerland (1997).

2. Mathur, N.K. \& Narang, C. K. - J. Chem. Educ., 67(11), p.938 (1990).

3. Raymond, L.; Morin, F.G. \& Marchessault, R.H. Carbohydr. Res., 246, p.331 (1993).

4. Brugnerotto, J.; Lizardi, J.; Goycoolea, W.; Argüelles-Monal; Desbrières, J. \& Rinaudo, M. - Polym., 42(8), p.3569 (2001).

5. Hirai, A.; Odani, H. \& Nakajima, A. - Polym. Bull., 26(1), p.87 (1991).

6. Niola, F.; Basora, N.; Chornet, E \& Vidal, P.F. - Carbohydr. Res., 238, p.1 (1993).

7. Roberts, G. A. F. - "Chitin chemistry”, The Mucmillan Press Ltd, London (1992).

8. Alonso, I.G.; Peniche-Covas, C \& Nieto, J.M. - J. Thermal Anal., 28, p.189 (1983).

9. Rinaudo, M.; Pavlov, G. \& Desbriéres, J. - Polym., 40, p.7029 (1999).
10. Peter, M.G. - J. Macromol. Sci-Pure \& Appl. Chem., 32A, p.629 (1995).

11. Signini, R \& Campana Filho, S.P. - Polímeros: Ciênc.Tecnol., 11(2), p.58 (2001).

12. Signini, R \& Campana Filho, S.P. - Polímeros: Ciênc. Tecnol., 4(4), p.63 (1998).

13. Varum, K.M.; Ottoy, M.H. \& Smidsrod, O. - Carbohyd. Polym., 46, p.89 (2001).

14. Rinaudo, M.; Desbrières, J.; Dung, P. Lê.; Binh Thuy, P.; \& Dong, N.T. - Carbohyd. Polym, 46, p.339 (2001).

15. Huggins, M.L. - J. Am. Chem. Soc. 64, p.2716 (1942).

16. Rinaudo, M.; Milas, M. \& Dung, P.L. - Int. J. Biol. Macromol., 15, p.281 (1993).

17. Cavalheiro, E. T. G.; Ionashiro, M.; Breviglieri, S. T.; Marino, G. \& Chierice, G. O. - Quím. Nova., 18, p.305 (1995).

18. Sreenivasan, K. - Polym. Degrad. Stabil., 52, p.85 (1996).

19. Hatakeyama, T \& Quinn, F.X. - "Thermal analysis: fundamentals and applications to polymer science", John Wiley \& Sons Ltd. Chichester (1999). 